\title{
Antibiotic resistance changing management of urinary tract infections in aged care
}

In the lead up to the global Antibiotic Awareness Week (16-22 November), it is timely to focus on the fact that bacterial infections that were once easily cured with antibiotics, such as those affecting the urinary tract, are becoming harder to treat.

Urinary tract infections (UTIs) are common in adults, and are prevalent in both hospital and community settings.

Escherichia coli bacteria - responsible for $80 \%-90 \%$ of uncomplicated UTIs - can display multidrug resistance.

UTIs can result in significant symptoms. When these are present, antibiotic treatment is typically indicated. However, for many people, including those in residential aged care facilities, asymptomatic bacteriuria has not been shown to be harmful. For this reason, routine testing for, or treatment of, asymptomatic UTIs in residential aged care facilities is not beneficial, except in catheterised patients at risk of complications, such as those with neutropenia.

Promoting the appropriate use of antibiotics for UTIs is an important consideration for residents in aged care facilities as excessive use - in the context of asymptomatic bacteriuria - may be contributing to the high prevalence of multidrug-resistant $E$. coli seen in this group.
In 2012, NPS MedicineWise began a 5-year campaign to reduce antibiotic prescribing in Australia, to bring it in line with the OECD (Organisation for Economic Co-operation and Development) average. The campaign encourages all Australians to use antibiotics responsibly. For health professionals, this means the judicious use and prescribing of antibiotics, and adherence to the principles of antimicrobial stewardship.

Health professionals can consolidate their knowledge on treating UTIs and minimising antibiotic resistance, and brush up on current guidelines and practices at www.nps. org.au/utis.

Lynn M Weekes

NPS MedicineWise, Sydney, NSW

Iweekes@nps.org.au 